\title{
STRUCTURES IN THE SPACE OF PLATONIC AND ARCHIMEDEAN SOLIDS
}

\author{
A B S S T R A C
}

This paper gives a classification of Platonic and Archimedean solids related to more-dimensional cubes. A general infinite net structure for space-filling mosaics created from the above solids has been derived and the node elements for grid structures based on our groups of solids have been described within the frame of constructive geometry. The topic is related to arts and design and raises further geometrical questions. 


\section{INTRODUCTION}

Platonic solid means a regular convex polyhedron. In each vertex of these polyhedra congruent regular polygons meet. Archimedean solids are semiregular convex polyhedra. The same combination of different types of congruent regular polygons meet in their vertices but, due to symmetry properties, the prisms and antiprisms are excluded from this group. It is natural that the sum of the angles between edges joining a vertex must be less than 360 degrees on all convex polyhedra. Based on these rules, we can compose the groups of the above solids and their description may be possible by sequences of numbers of the edges of regular polygons meeting in a vertex. For instance, 4.4.4 means the cube and 5.6.6 the truncated icosahedron, the polyhedral basic form of the football.

These solids can be classified by the number of their edges having different spatial stands. There is one polyhedron in every group which is the hull of a possible three-dimensional model of a more-dimensional cube. (We have however two special Archimedean solids that are not members of these groups from our points of view.)

The 3-dimensional model (3-model) of a $k$-dimensional cube ( $k$-cube) may be generated by the well known procedure of moving the lower-dimensional elements along the edges parallel to the direction of the next dimension. This procedure is easy to follow from a vertex through edge, face and cube and following up to the four- and further more-dimensional cubes. (The 3-dimensional model of a three-dimensional element from a more-dimensional cube can be a parallelepiped as well.) The model has $2^{k}$ vertices and $k$ groups of parallel edges. The number of edges that join each vertex is always $k$. We can even get multiple coinciding vertices and we may demand that the longitude of the edges be equal. The properties of our three cube-models fitting inside of Archimedean solids and their connections to the structure of the regular and semi-regular solids will be studied.

Branko Grünbaum, in his paper Uniform tilings of 3-space [2], enumerated the possible space-filling periodical tessellations (further shortened as tiling) based on the Platonic and Archimedean solids as well. The structures of point nets which could be basic patterns holding vertices of these tilings are asked for and also geometrical models of node elements of spatial grid structures based on our groups of solids are constructed and in the end these are unified in a common form. 


\section{SPECIAL SETS OF PLATONIC AND ARCHIMEDEAN SOLIDS}

These solids can be ordered into sets by the number of their edges having different spatial stands. There is one polyhedron in all groups which is the hull of a possible three-dimensional model of a more-dimensional cube and some members of the groups can constitute the geometrical basis of a node element connecting to the bars of spatial grid structures.

\section{GROUP 3}

Our first group is the basis and has only one member, the normal cube i.e. the Platonic hexahedron (4.4.4). This polyhedron has 12 edges and they have 3 different spatial stands. That is the reason why this set is called group 3 . The edges are perpendicular to the faces of the cube. Thus this solid itself can be a node element for a spatial grid structure constructed from cubes' edges. The 3-dimensional model of this solid can also be a cube. For the sake of completeness, it has to be stated that this solid is the part of itself and their vertices also coincide with themselves. The layers of the vertices in the spacefilling mosaic of cubes have a pattern of squares and these can be projected to each other perpendicularly to the parallel planes of the vertices' layers.

\section{GROUP 6}

The edges of the next polyhedra have 6 different spatial stands: Platonic tetrahedron (3.3.3) and octahedron (3.3.3.3) as well as the Archimedean truncated tetrahedron (3.6.6), cuboctahedron (3.4.3.4) and truncated octahedron (4.6.6) (Figure 1).

Taking 6 starting edges, parallel with differently oriented edges of these solids, we can construct a 3-dimensional model of the 6-dimensional cube based on the method described in the introduction (Figure 2). The hull of this model is the truncated octahedron. This solid is a common part of a cube and an octahedron (Figure 3a) and the inner vertices of the 6-cube's 3-model join the vertices of two solids similar to the above ones (Figure $3 b$ ). Based on some examples, it can be seen in Figure 4 that the elements of the above 2 Platonic and 2 Archimedean solids, members of the group 6, join the parts of our 6-cube's 3-model.

The vertices of this model fit onto parallel planes and constitute planar nets of squares. The nets are moved with a half of a diagonal of the squares on the planes following each other. The distance of the vertices is 2 if the distance 


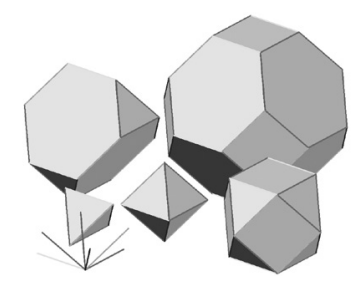

Figure 1

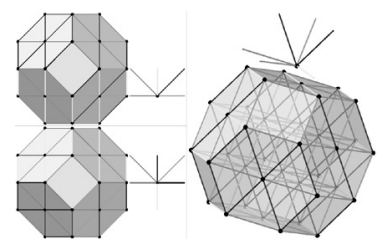

Figure 2

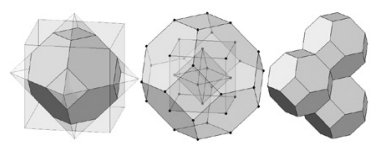

Figure $3 a / 3 b / 3 c$

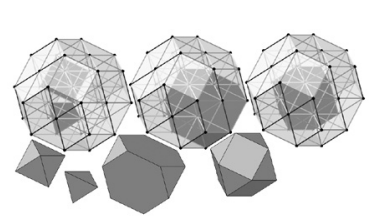

Figure 4

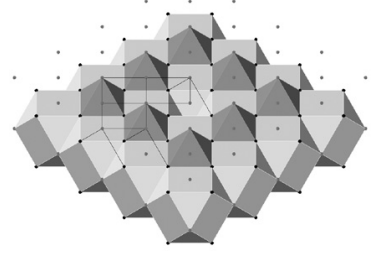

Figure 5

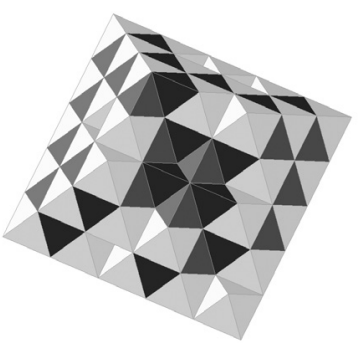

Figure 6

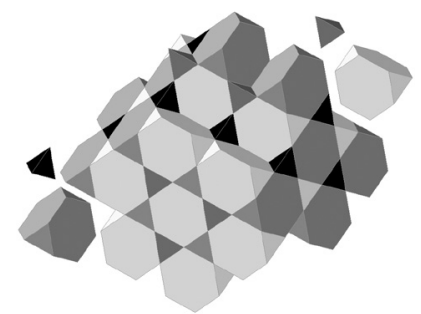

Figure 7

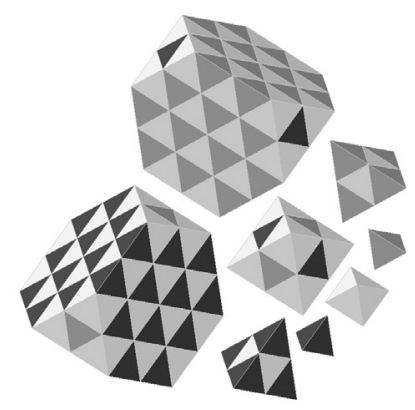

Figure 8

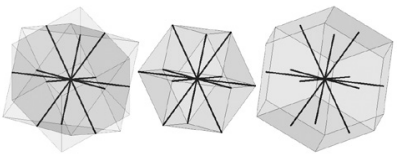

Figure $9 a / 9 b / 9 c$

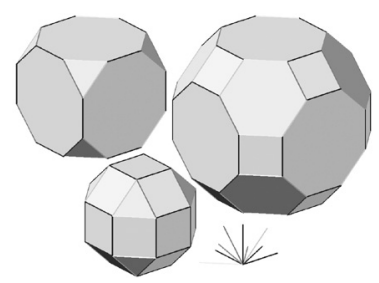

Figure 10

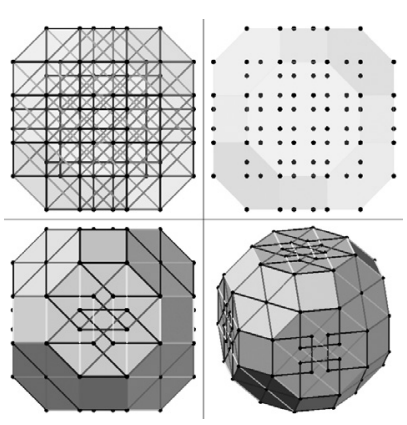

Figure 11 
of the vertices' layers is $2^{1 / 2}$. This structure of nets is the same in 3 directions perpendicular to each other. It is well known that the space can be filled with the truncated octahedron (Figure 3c) and this means that it can be done with our 6-cube's 3-model as well. The vertices inside this spatial mosaic build the same set of nets like the one above. As it is well known, $2^{3}$ cubes are needed, attached with faces to each other, to double the edges' length of a cube and $m^{3} 3$-cubes are needed to $m$-tuplicate the edges' length. Generally, $m^{k} k$-cubes are needed to $m$-tuplicate the edges' length of a $k$-dimensional cube [7]. This construction may be described as moving the cubes along the edges and in case of our 6-cube's 3-model the vertices obtained constitute the above net structure of squares.

Based on the former interrelations, it can be stated that the tessellations composed from the solids of the group 6 fit into the nets of squares described above. Figure 5, for instance, shows the periodical space-filling mosaic of solids (3.3.3.3) and (3.4.3.4) in our net structure. Probably the most frequently used lattice girder is based on mosaic of tetrahedra and octahedra (Figure 6) and the periodical tessellation of tetrahedra and truncated tetrahedral are presented as well in Figure 7. These spatial mosaics may have fractal structure because their elements can be composed from tetrahedra and octahedra (Figure 8). The vertices of these mosaics also join the above nets of squares.

To construct spatial grid structures, the initial ray group of the edges in a centrally symmetric shape needs to be formed and 12 rays are gained. These are perpendicular to the edges of a cube and/or to those of an octahedron. These polyhedra are dual pairs of each other (Figure 9a). The basic bars joining the center can be arranged as a common initial point and the vertices of a cuboctahedron as well (Figure 9b). But, from practical point of view, the node element could be a rhombic dodecahedron the faces of which are perpendicular to the 12 basic rays (Figure 9c).

\section{GROUP 9}

The edges of the next polyhedra have 9 different spatial stands: Archimedean truncated cube (3.8.8), (small) rhombicuboctahedron (3.4.4.4) and great rhombicuboctahedron or truncated cuboctahedron (4.6.8) (Figure 10). The same set of basic edges from Platonic solids can be obtained if a tetrahedron (or the dual pair of two ones) is constructed into a cube or if the dual pair of an octahedron and a cube is taken. 


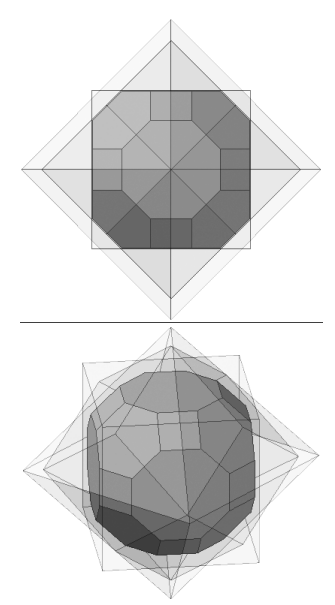

Figure 12

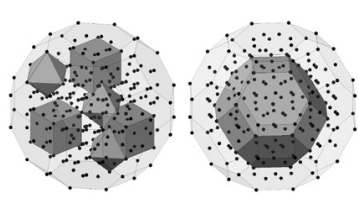

Figure 13a Figure 13b
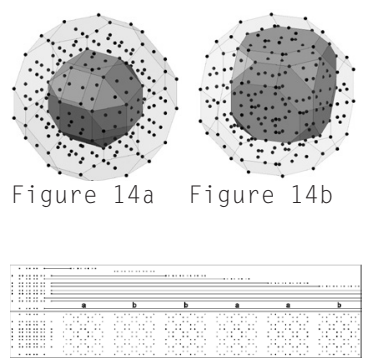

Figure 15
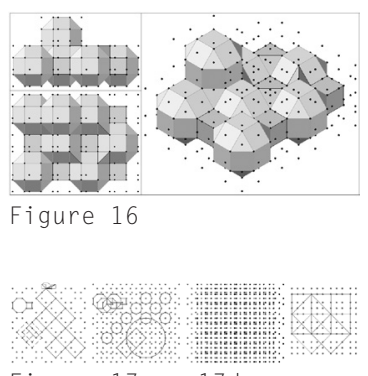

Figure 17a - 17d

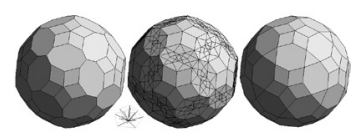

Figure $17 a-17 d$

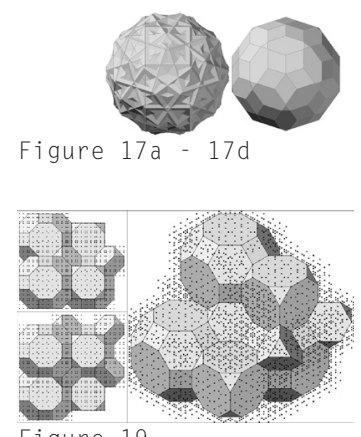

Figure 19

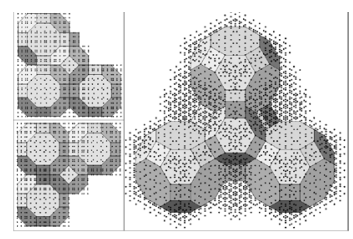

Figure 20

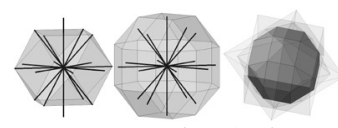

Figure 21a / 21b / 21c

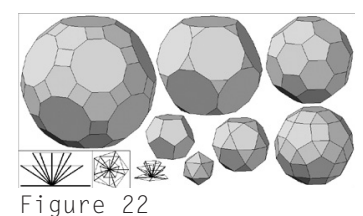

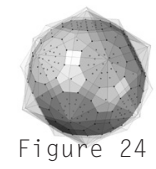

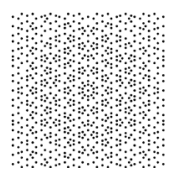

Figure 25a Figure 25b 
Gathering 9 starting edges, parallel with the differently oriented edges of these solids, a 3-dimensional model of the 9-dimensional cube can be constructed based on the method described in the introduction (Figure 11). The hull of this model is the truncated cuboctahedron. This solid is the common part of a cube, an octahedron and a rhombdodecahedron (Figure 12). The last solid is similar to the node element of the group 6. The inner vertices of the 9-cube's 3 -model join the vertices of cubes and octahedrons as well (Figure 13a). It may be seen in Figure 13b that the elements of the 6-cube's 3-model join those of the 9-cube. Thus the members of the group 6 fit into the 9-cube's 3-model. The set of the group 9 holds the sets of the groups 3 and 6: ((group 3) (group 6) group 9). It can be seen in Figures 14a-b that the elements of Archimedean solid-members of the group 9 also join the parts of the 9-cube's 3-model.

The vertices of our model fit onto parallel planes. It is indicated by these planar patterns that the nets of the vertices are completed (Figure 15 front and top views: the vertices of the 9-cube's 3-model are the black points, the additional nodes are the gray ones). With an additional layer (Figure 15 front view) the following structure is obtained: the $a$ and $b$ nets of points are vertices of congruent planar Archimedean tessellations of squares and octagons (4.8.8). Pairs of two $a$ and two $b$ nets follow each other. The distance between the pairs is 2 , the distance between neighboring $a$ and $b$ nets is $2^{1 / 2}$. These nets are shifted with $2^{1 / 2}$ related to each other and the distance of adjoining vertices is 2 inside the same nets (Figure 15). The vertices of the space-filling periodical mosaic of solids (4.4.4), (3.3.3) and (3.4.4.4) join the nodes of the above net structure (Figure 16: front and top view as well isometric orthogonal axonometric projection). The nets of attached squares can be constructed inside above $a$ and $b$ nets (Figure 17a). Thus the vertices of mosaics created from members of group 6 can join nodes of our new structure of nets. The scaled structures of the above nets can be marked inside our infinite point structure. Thus from this point of view, the former tilings are fractals.

The doubling of the edge length of our 9-cube's 3-model generates a new pattern of vertices. The whole net of nodes can be descibed in the following way as well: the above congruent $a$ and $b$ nets are parallel with three planes perpendicular to each other. These nets must be copied in their own planes in directions of three vectors determined by nodes of the basic net. The last construction and the newer planar pattern are shown in Figure 17a-b. Figure $17 \mathrm{c}$ shows the new, shifted $a$ and $b$ nets together which are signed with gray and black points, respectively . It is again easy to see that spatial net structures of attached squares fit into our new point-net structure. 
Based on all interrelations described up till now, it can be stated that all the possible 11 space-filling mosaics of Platonic and Archimedean solids fit into this structure. But, it has to be remarked: if the edge length of the solids is 1 , it must be shortened to $\left(2^{1 / 2}-1\right)$ in case of tiling 9 and to $\left(2-2^{1 / 2}\right)$ in cases of tilings 7 and 10 (Figure 17a and Table 1). Tiling 1, tessellation of cubes, can be created with different edge lengths but not with 1 .

In cases of tilings 7 and 10, the layers of nodes may be erased. The thinned planar pattern is shown in Figure 17d. The distance of the layers of three congruent planar point nets is $\left(2^{1 / 2}-1\right)$ equal to the edge length of the solids and the distance of the layers' triplets is $\left(2-2^{1 / 2}\right)$. Thus the proportion of the distances is $1: 2^{1 / 2}$. The nets of attached squares can be constructed onto the layers and these are not shifted related to each other (Figure 17d). Thus the vertices of cubes' tiling join nodes of our thinned net structure. However, a wider and properly shifted net structure may be chosen and, in this way, the vertices of tilings created with solids of the group 6 can join the points of this structure as well (Figure 17d: black and gray, oblique squares). Fractals are also imaginable in the above sense.

The next Table 1 shows fitting of the possible tilings, built from Platonic and Archimedean solids, into the net structure derived from the multiplied 9-cube's 3-model. One may see in Figures 19 and 20 the tilings 8 and 11 with points of the net structure in front and top view as well as in isometric orthogonal axonometric projection, as examples.

\begin{tabular}{|c|c|c|c|c|c|c|c|c|c|c|c|}
\hline$k$-cube & 3 & \multicolumn{5}{|c|}{6} & \multicolumn{3}{|c|}{9} & \multicolumn{2}{|l|}{$k$-cube } \\
\hline $\begin{array}{l}\text { solid / } \\
\text { tiling \# }\end{array}$ & 4.4 .4 & 3.3 .3 & 3.6 .6 & 3.3.3.3 & 3.4 .3 .4 & 4.6.6 & 3.8 .8 & 3.4.4.4 & 4.6 .8 & $\begin{array}{l}\text { solid / } \\
\text { tiling \# }\end{array}$ & $k$-cube \\
\hline 1. & + & & & & & & & & & $+<$ & 3 \\
\hline 2. & & + & & + & & & & & & + & \multirow{5}{*}{6} \\
\hline 3. & & + & + & & & & & & & + & \\
\hline 4. & & & & + & + & & & & & + & \\
\hline 5. & & & & & & + & & & & + & \\
\hline 6. & & & + & & + & + & & & & + & \\
\hline 7. & & & & + & & & + & & & $>+$ & \multirow{2}{*}{$6 \& 9$} \\
\hline 8. & & & + & & & & + & & + & + & \\
\hline 9. & + & + & & & & & & + & & $>>+$ & \multirow{3}{*}{$3 \& 6 \& 9$} \\
\hline 10. & + & & & & + & & & + & & $>+$ & \\
\hline 11. & + & & & & & + & & & + & + & \\
\hline$k$-cube & 3 & \multicolumn{5}{|c|}{6} & \multicolumn{3}{|c|}{9} & \multicolumn{2}{|l|}{$k$-cube } \\
\hline
\end{tabular}


To construct spatial grid structures, the initial ray group of the edges needs to be formed into a centrally symmetric shape and 18 rays are obtained. The 12 basic bars joining the center can be arranged, as a common initial point, and the vertices of a cuboctahedron as well and the remaining 6 ones are perpendicular to the square faces of the solid (Figure 21a). But, from practical point of view, a better node element could be a small rhombicuboctahedron whose square faces are perpendicular to the 18 basic rays (Figure 21b). This solid is also the common part of a cube of an octahedron and a rhombdodecahedron (Figure 21c).

\section{GROUP 15}

The edges of the next polyhedra have 15 different spatial stands: Platonic dodecahedron (5.5.5) and icosahedron (3.3.3.3.3) as well as the Archimedean icosidodecahedron (3.5.3.5), truncated dodecahedron (3.10.10), truncated icosahedron (5.6.6), rhombicosidodecahedron (3.4.5.4) and truncated icosidodecahedron (4.6.10) (Figure 22).

Gathering 15 starting edges, parallel with the differently oriented edges of these solids and joining a common point, a 3-dimensional model of the 15-dimensional cube can be constructed based on the method described in the introduction. The hull of this model is the truncated icosidodecahedron (Figure 23). This solid is the common part of a dodecahedron an icosahedron and a rhombic triakontahedron (Figure 24). The last one is created from 30 congruent rhombus faces whose diagonals are in proportion of the golden mean.

The tilings cannot be built only with the solids from this group. The numbers of the vertices and edges of the 15-cube are already very big (32768 and 245760, respectively) to handle these elements with a common computer processing the above described operations. (The doubling of the 9-cube's 3-model created $2^{9} \times 2^{9}=262144$ vertices. This construction in case of the 15-cube's 3-model could result in $2^{15} \times 2^{15}=1073741824$ vertices.) However, one can imagine that the elements of this group's members join those of the 15-cube's 3-model as well as that relevant planar nets are parallel with faces of a dodecahedron and their distances are combinations of different values following each other in proportion of the golden mean. Figures $25 \mathrm{a}$ and $25 \mathrm{~b}$ show a partial set of the models vertices and some relevant layers of those.

The centrally symmetric initial ray group holds 30 rays. These are perpendicular to the square faces of the rhombicosidodecahedron (3.4.5.4). This solid is also the common part of a dodecahedron an icosahedron and a rhombic triakontahedron (Figure 26). 


\section{COMMON GROUP 21 AND NODE ELEMENT}

It is known that five cubes can be constructed inside a dodecahedron. Their vertices join each other and the cubes' edges are parallel with those of the dodecahedron. Three of the nine basic edges in the group 9 are parallel with the cube's edges. Thus the basic edges of the group 15 and ((group3) (group 6) group 9) can be stated from a common point in a way that 3 common edges are obtained. There are 21 differently oriented rays parallel with the edges of the solids which are members of our former 4 groups. Thus the above solids can create the group 21 in this form: (((group 3) (group 6) group 9) ((group 3) (group 15) group 21).

The centrally symmetrical shape of the basic ray group holds 90 rays. Figure 27 a shows the solid whose 60 rhombic and 30 symmetrical octagonal faces are perpendicular to these rays and their center points join them. This is no more an Archimedean solid but the hull of a 16-cube's 3-model (Figure 27b). (Please read on the matter of principle in [1] and [5]). With a possible correction 20 new faces (that means 20 new initial rays) are obtained which are parallel with the faces of an icosahedron (Figure 27c). If a rhombicuboctahedron (our node element in the group 9) is fitted into a cube and they are five times fitted in a dodecahedron, a solid can also be obtained the faces of which are parallel with those of the last one (Figure 28). Thus both above solids can be constructed from the common part of 5 rhombicuboctahedron by parallel and centrally symmetric moving of the resulting faces.

\section{Archimedean solids outside our groups}

The snub cube (3.3.3.3.4) and the snub dodecahedron (3.3.3.3.5) have 48 and 122 differently oriented edges out of altogether 60 and 150 ones, respectively. Both have a symmetrical, so called chiral pair which are rotated in the opposite direction. However the pairs together can be constructed from 50 and 130 differently oriented edges, respectively.

\section{CONCLUDING REMARKS}

Our topic raises further geometrical questions especially on the field of group 15. For instance, could a space-filling periodical mosaics be constructed from the solids of group 15 if lower-dimensional parts of the truncated icosidodecahedron - our 3-model of the 15-cube - are used as well? How can the patterns of the points in the net structure belonging to group 15 help to construct space-filling periodical mosaics based on the 3-model of the 5-and 
10-cube hulled with rhombic faces [8]? It may herein be mentioned that the rhombicosidodecahedron (3.4.5.4) is the node element of the geometrical toy and modeling tool, Zometool also used in scientific research.

As mentioned before, the geometrical interrelations of the described tilings can be used for construction of spatial lattice girders. The Platonic and Archimedean solids are basic cells of special buildings in several architectural competitions and for example of exhibition pavilions. Sets of derived solids are employed even in the special field of design of space stations as well [3, 4]. This topic can raise probably further thoughts for the technical design.

The 2-dimensional shadows of the models and the intersections of the spacefilling mosaics with planes allow unlimited possibilities to produce periodical symmetric plane-tiling [8]. These and the 3-models build the base of several works of art and aid industrial design. Moving intersectional planes create series of tessellations or grid-patterns transforming into each other which can be shown in various animations [9]. These provide possibilities for exhibitions, publicity work and further usage.

The creation of the constructions and figures for this paper was aided by the AutoCAD ${ }^{\circledR}$ program and Autolisp routines developed by the author. 
Vörös, László "Planar tessellations based on shadows of more-dimensional cubes" Paper presented at the 25th National and 2nd International Scientific Conference, monGEometria, Belgrade, Serbia, June 24-27, 2010. Proceedings-CD, ISBN 978-86-7924-038-5, paper Vörös, László “Sets of Space-filling Zonotopes with Connection to Art and Design" Paper presented at the 14th International Conference on Geometry and Graphics, ICGG, Kyoto, Japan, August 5-9, 2010. Proceedings-DVD, ISBN 978-4-9900967-1-7, extended abstract and full paper, 231

http://epitesz.pmmk.pte.hu/tervezesi_es_epiteszeti_ismeretek_tanszek/oktatok_tervezes/voros_ laszlo/voros_laszlo_videok

http://en.wikipedia.org/wiki/Convex_uniform_honeycomb

N.B . The creation of the constructions and figures for this paper was aided by the AutoCAD ${ }^{\circ}$ program and Autolisp routines developed by the author. 